CLAWAR 2018: 21st International Conference on Climbing and Walking Robots and the Support Technologies for Mobile Machines, Panama City, Panama, 10-12 September 2018

\title{
NAO ROBOT AS A DOMESTIC ROBOT
}

\author{
JESSICA P. M. VITAL \\ INESC TEC-INESC Technology and Science and \\ University of Tras-os-Montes and Alto Douro, Vila Real, Portugal \\ Email:Jessicavital_hotmail.com \\ ORCID: 0000-0002-1660-7718 \\ N. M. FONSECA FERREIRA \\ INESC TEC - INESC Technology and Science and \\ Institute of Engineering Of Coimbra, Polytechnic Institute of Coimbra, and \\ Knowledge Research Group on Intelligent Engineering and Computing for Advanced \\ Innovation and Development (GECAD) of the ISEP/IPP, Portugal. \\ Email: nunomig@isec.pt \\ ORCID: 0000-0002-2204-6339 \\ ANTONIO VALENTE \\ INESC TEC-INESC Technology and Science and \\ University of Tras-os-Montes and Alto Douro, Vila Real, Portugal \\ Email: avalente@utad.pt \\ ORCID: 0000-0002-5798-1298

\begin{abstract}
Over the years robotics has made great progress. Nowadays, robots begin to be part of the life of any person, designated social robotic. Humanoid robots are fascinating and have several advantages, such as they can work in places where there is a risk of contamination, risk of health, danger of life, places that are difficult to access. They also are able to access different types of terrain and to climb stairs. NAO robot is currently the humanoid platform with high sensory capacity that it has lower costs in the market. This robot is similar to human in order to have a more real and natural with society. Using the capabilities of the robot and adding other sensors, we can have a more powerful machine in our society. NAO robot is presented in this paper as a domestic robot.

Keywords: NAO robot, wireless sensors, Humanoid robots, Domestic robots, object recognition
\end{abstract}

\section{Introduction}

Over the years robotics has made great progress, nowadays robots begin to be part of the life of any person, designated social robotics [1].

Humanoid robots are fascinating and have several advantages, such as they can work in places where there is a risk of contamination, risk of health, danger 
of life, places that are difficult to access [2]. They also are able to access different types of terrain and to climb stairs [3]. The behavior of humanoid robots produces feelings that facilitate the communication between machine and man.

There are many cases where you need a machine similar to the man to be able to replace it in your work environment [4].

In recent years, social robotic has seen the emergence of sophisticated humanoid robots, as Honda Asimo and NAO [5].

We chose the platform NAO that is currently the humanoid platform with high sensory capacity that it has lower costs in the market. This robot is similar to the human in order to have a more real and natural interaction with society. NAO is a good platform for software development because it is easy to learn how to program and it has a high sensory ability.

This paper is divided into four sections. The first section gives a brief introduction of different domestic robots. The second section presents NAO robot. An extension of platform NAO using sensors and an architecture of recognize objects using NAO robot is described in third section. Our conclusions are drawn in the final section.

\section{Domestic Robots}

Domestic Robots can help you carry out all kinds of tasks at home, such as cooking, cleaning, company, babysitting or answering questions. Science fiction has come to fruition, and they are available to make people's lives easier by dismissing people from household chores and giving them the opportunity to do other leisurely things [6].

Nowadays all people use domestic robots in their homes, such as:

- Roomba Vacuum: can vacuum the floor of the house alone [7];

- Bimby: can cook alone meals, sweets or whatever the person wants;

- Winbot: robot that clean the glass;

- Husqvarna automower: robot that mows the lawn very efficiently.

The area of robots has been an area of great evolution appearing also robots as: Zenbo, Kuri. The use of humanoid robots as domestic robots were appeared, as, Pepper [8], Honda Asimo and NAO robot [5]. These robots are more developed able to do different household tasks, thus becoming much more expensive when compared with the simpler robots and that only perform one task. To combat this situation we present the NAO robot, as a domestic robot. 


\section{NAO robot}

NAO robot (figure 1) is humanoid robot developed by Aldebaran Robotics, a French company. It has $58 \mathrm{~cm}$ in height and it weighs $5.4 \mathrm{~kg}$ which make it easy to transport [9]. With twenty five degrees-of- freedom (DOF) and more than 50 sensors, NAO is a robust robot to develop a wide range of applications.

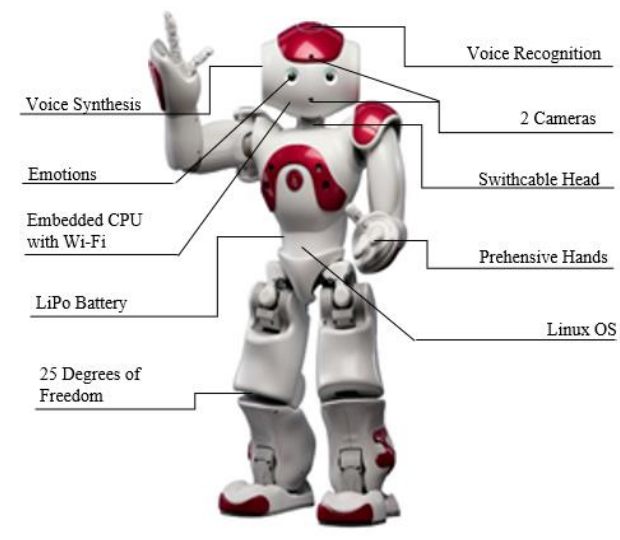

Figure 1- NAO robot

NAO is specially used as a social robot. NAO robot has a similar appearance when compared with a human. NAO allows to perform several basic actions, such as sitting, walking, picking up objects with your hands, among others.

NAO robot has a disadvantage, its autonomy. In normal use NAO just has 90 minutes of autonomy which makes this robot only used as proof of concept and not as a daily instinctive use robot. On the other hand we can conclude that the robot has several advantages, as it is easy to carry, because it is small and it is light. It has equipped with two cameras, like human eyes and can speak and can recognize the most popular languages in world [10]. Are so many applications that NAO robot has been used, such as: sports and interaction with humans.

\section{A. Rehabilitation}

By cameras robot can see the movement of patients and avoid them if the movement are correct or no [11]. NAO is able to replicate the movement of humans, so it can explain to patient how to the correct movement [12,13].

B. Autism Children

NAO robot has been used to help autistic children in improving their behavior $[15,16]$. The studied cases show that children suppress the autistic behavior during human-robot interaction and they can maintain visual contact with NAO [17]. 


\section{Traditional Robotic Soccer}

NAO robot participate in RoboCup (the World Championship of Robotics) as a soccer playing NAO robot has been the star of the Standard Platform League where robots are expected to operate fully autonomously [17, 18].

\section{Nao robot as a domestic robot}

In this section we present different architectures that can reach NAO robot as a domestic robot. Firstly is presented an extension of NAO robot using sensors and after is presented an architecture to recognize objects.

\subsection{Platform using sensors}

This architecture present the integration of three sensors to NAO robot (figure 2). To integrate the sensory network were chosen the following sensors:

- Temperature sensor: LM35 sensor 545753 YWrobot.

- Photoresistor Light sensor: 531289 ywrobot;

- Gas sensor: MQ Series Gas Sensor Module L1te v1.0 sandbox electronics

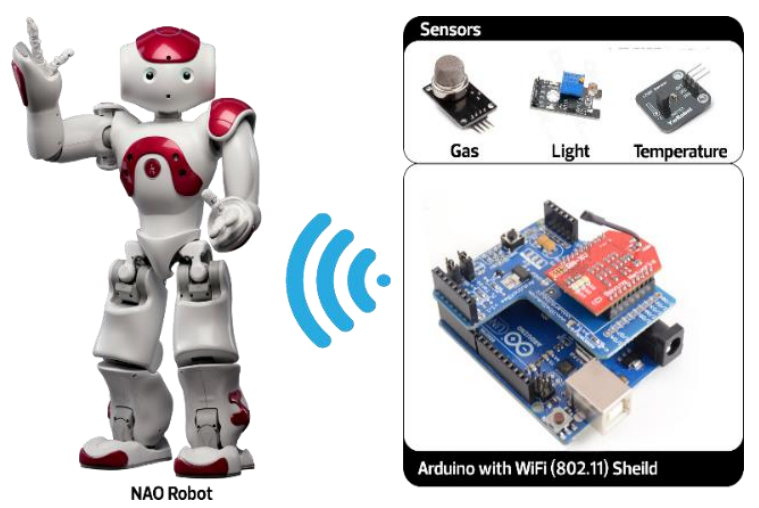

Figure 2- Architecture integrating three sensors to NAO robot

When integrating three sensors in Arduino, a set of collections is provided in order to validate their operation against the accuracy and precision.

The Arduino is equipped with the Wireless Proto Shield by using SPI Communication. This allows pairing both NAO and sensorial array using WIFI 802.11 [19]. Using Choreographe, the tool used to program the NAO, it is possible to elaborate a set of tasks for the interaction with the general public. 
An Arduino library called CoGasSensorShield was used. This library allowed to receive a value (e.g., gas sensor) via Wifi from an Arduino board and thus issue an alert when desired. Given the limitations of the library, it was extended in order to access the three sensors previously chosen, and with the respective intervals that had previously been analyzed. After this work of adaptation, a project was developed in Choregraphe that allowed to execute a set of movements and dialogues associated to the output of the program in Arduino in order to promote the human-machine interaction.

Intervals of values for the presence of gas, high temperature and luminosity were analyzed and elaborated. In the Arduino, an alert was sent via Wifi when values outside these ranges were registered. It reads and analyzes the values that the sensors transmit and throws an alert, if it justifies, and if it has in the presence of a dangerous situation. So that the alert is given correctly, different parameters have been stipulated. The Arduino continuously reads all sensor values and processes this information and whenever there is a state change it alerts the robot.

NAO robot will transmit alerts in different situations that was predefined, in situations of danger to people, taking into account the ambient temperature, gas levels and brightness. If the sensors detect that ambient has a high level of temperature and the light is less, the robot will transmit a fire alert. In case of an alert, NAO tells people to evacuate and explains to them what is happening. The alert is also given by sending a message directly to the responsible. In case of fire the message will be sent directly to the firemen, with the address, in order to solve the problem effectively and quickly.

\subsection{Recognition Objects}

Choreographe Software is the tool used to program the NAO, it is possible to elaborate a set of tasks for interaction with the general public. Through this program that we used to program NAO robot can learn the different objects so that later it can identify them. As proof of concept we used five types of object to recognize, such as, a banana, a bottle of water, a mobile phone and a medicine. Objects of different areas that are essential in ambient assisting living.

First we used robot to capture different images with different objects. We selected different objects in different positions and at different distances, in order to create a dataset, so that the robot could distinguish the different objects. Then the images were segmented. The contour of the object was made so that the robot could distinguish it when the object is surrounded by other objects. After the outline of each object, we indicate the name of the object and the position in which it is. 
All the information is sent to the robot so that it can memorize the different images, the name of the different objects and their position so that the robot can later distinguish the different objects in different circumstances.

Finally, the vision recognition module belonging to the Choreographe program was used. We was used this module robot acquire an image in real time and analyze, if him recognize they tell what it is.

One of the limitations of NAO robot is the memory, so as future work will be created a cloud computing service. It is through the cloud computing service that the robot can recognize objects more quickly and accurately. Cloud will have a wide set of images of many objects in different positions so that the robot can recognize all the existing objects in a more viable and faster way of processing. Despite being connected to the cloud will always have the ability to recognize a set of predefined objects, essences of that environment so that in case there is some failure of connection to the cloud the robot with him in the same way do their activities. In a house we may have one robot but we can have more than one.

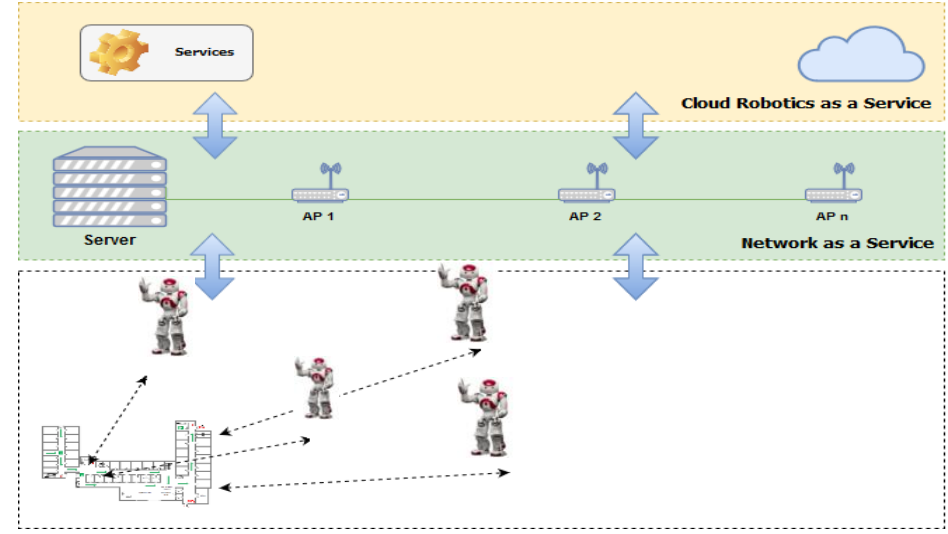

Figure 3- Cloud Operating Diagram

In figure 3 we can observe a cloud operating diagram. There we see robots in different places of the house communicating each other and communication with the server. The server communicating with the cloud to obtain the information and give it back to the robot.

\section{Conclusion}

NAO robot can perform different domestic tasks. NAO robot can walk, talk, see and grab objects. Despite its basic functions, through cameras the robot can recognize objects and people. Adding sensors to the robot, it is able to send 
environmental alerts in dangerous situations. We can conclude that the robot is a domestic robot capable of doing not only domestic tasks, but also doing company or having a dialogue. Having a humanoid aspect and an affordable cost is a key point in the acceptance of the robot by our society.

\section{Acknowledgments}

This work is financed by the ERDF - European Regional Development Fund through the Operational Programme for Competitiveness and Internationalization - COMPETE 2020 Program within project POCI-01-0145-FEDER-006961, and by National Funds through the FCT - Fundação para a Ciência e a Tecnologia (Portuguese Foundation for Science and Technology) as part of project UID/EEA/50014/2013.

\section{References}

1. T. Fong, I. Nourbakhsh and K. Dautenhahn, "A survey of socially interactive robots," Robotics and Autonomous Systems, pp. 143-166, 2003.

2. R. Calo, "Robots and privacy," 2010.

3. A. Hornung, K. M. Wurm and M. Bennewitz, "Humanoid Robot Localization in Complex Indoor Environments," in Intelligent Robots and Systems (IROS), 2010.

4. L. Takayama, W. Ju and C. Nass, "Beyond Dirty, Dangerous and Dull: What Everyday People Think Robots Should Do," in Proceedings of the 3 rd ACM/IEEE international conference on Human robot interaction, 2008.

5. I. Rodriguez, A. Astigarraga, E. Jauregi, T. Ruiz and E. Lazkano, "Humanizing NAO robot teleoperation using ROS," in 14th IEEE-RAS International Conference on Humanoid Robots (Humanoids), 2014.

6. J. Carpenter, J. M. Davis, N. Erwin-Stewart, T. R. Lee, J. D. Bransford and N. Vye, "Gender Representation and Humanoid Robots Designed," International Journal of Social Robotics, vol. 1, pp. 261-265, 2009.

7. J. Forlizzi and C. DiSalvo, "Service robots in the domestic environment: a study of the roomba vacuum in the home," in Proceedings of the 1st ACM SIGCHI/SIGART conference on Human-robot interaction, 2006.

8. J. Lafaye, D. Gouaillier and P.-. B. Wieber, "Linear Model Predictive Control of the locomotion of Pepper,a humanoid robot with omnideirection wheels," in Humanoid Robots (Humanoids), 2014 14th IEEE-RAS International Conference on, 2014. 
9. D. Gouaillier, V. Hugel and P. Blazevic, "Mechatronic design of NAO humanoid," in Robotics and Automation, 2009.

10. S. Fojtu, M. Bresler and D. Prusa, "Nao Robot Navigation Based on a Single VGA Camera," in 17th Computer Vision Winter Workshop, 2012.

11. D. L. Recio, E. M. Segura, L. M. Segura and A. Waern, "The NAO models for the elderly," in Proceedings of the 8th ACM/IEEE international conference on Human-robot interaction, 2013.

12. C. Stanton, A. Bogdanovych and E. Ratanasena, "Teleoperation of a humanoid robot using full-body motion capture, example movements, and machine learning," in Proceedings of Australasian Conference on Robotics and Automation, 2012.

13. J. Lei, M. Song, Z.-N. Li and C. Chen, "Whole-body humanoid robot imitation with pose similarity evalution," Signal Processing, vol. 108, pp. 136-146, 2015.

14. S. Shamsuddin, H. Yussof, L. I. Ismail, S. Mohamed, F. A. Hanapiah and N. I. Zahari, "Initial Response in HRI- a Case Study on Evaluation of Child with Autism Spectrum Disorders Interacting with a Humanoid Robot NAO," International Symposium on Robotics and Intelligent Sensors, pp. 1448-1455, 2012.

15. S. Shamsuddin, H. Yussof, L. I. Ismail, S. Mohamed, F. A. Hanapiah and N. I. Zahari, "Humanoid Robot NAO Interacting with Autistic Children of Moderately Impaired Intelligence to Augment Communication Skills," International Symposium on Robotics and Intelligent Sensors, pp. 1533 1538, 2012.

16. A. Tapus, A. Peca, A. Aly, C. Pop, L. Jisa, S. Pintea, A. Rusu and D. David, "Children with autism social engagement in interaction with Nao, an imitative robot : A series of single case experiments.," Interaction studies, pp. 315-347, 2012.

17. T. Niemuller, A. Ferrein, G. Eckel, D. Pirro, P. Podbregar, T. Kellner, C. Rath and G. Steinbauer, "Providing Ground-truth Data for the NAO Robot Paltform," RoboCup, pp. 133-144, 2010.

18. L. M. Cruz, Humanoid Robot NAO: Developing Behaviors for Soccer Humanoid Robots, LAP Lambert Academic Publishing, 2013.

19. J. P. M. Vital, N. M. M. Rodrigues, M. S. Couceiro, C. M. Figueiredo and N. M. F. Ferreira, "Fostering the NAO platform as an elderly care robot," in Serious Games and Applications for Health (SeGAH), 2013. 